\title{
Canina fossa abscess and treatment
}

\author{
Muhammad H. Chandha*
}

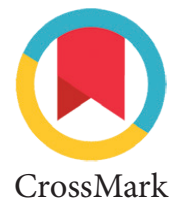

\section{Abstract}

Objective: Canina fossa abscesses are rarely reported in the literature. Tooth infection is often derived from bacteria associated with the abscess. Canina fossa abscesses can lead to the development of secondary abscesses, infection of incisors and canines in the upper jaw and maxillary sinusitis infection.

Methods: Odontogenic infections can easily spread throughout the muscles and the face, which can cause abscesses mucolabial or accumulation of pus in the eyelid and face.
Results: Patients experienced a history of pain and fever. Physical examination showed enlargement and swelling of the right cheek and panoramic radiographs appear radiolucent on the apex of tooth tip 13. Treatment included an intraoral drainage incision at the apex of the tooth 13 and continued administration of antibiotic therapy.

Conclusion: Successful treatments require early recognition, determination of etiological factors, appropriate medical and surgical management.
Department of Oral Surgery and Maxillofacial, Faculty of Dentistry, Hasanuddin University, Makassar, Indonesia
*Corresponding to: Muhammad H. Chandha, Department of Oral Surgery and Maxillofacial, Faculty of Dentistry, Hasanuddin University, Makassar, Indonesia muhencha@gmail.com

Received: 31 0ctober 2017 Revised: 23 November 2017 Accepted: 14 January 2018 Available online: 1 April 2018
Keywords: Odontogenic Infections, Fossa Canina abscess

Cite this Article: Chandha MH. 2018. Canina fossa abscess and treatment. Journal of Dentomaxillofacial Science 3(1): 54-57. D0I: 10.15562/ jdmfs.v3i1.637

\section{Introduction}

The oral cavity is a breeding ground for various microorganisms where injury can occur inevitably ${ }^{1}$ microorganisms normally present in the oral cavity may cause infection if; firstly, the nature of the microorganism is altered both in quality and quantity; secondly, bacteria penetrates the oral mucosa and tooth pulp; and thirdly, the immune system and cellular defenses are disrupted. Any combination of the above incidents can result in acute or chronic infections. The acute condition is usually accompanied by severe swelling and pain with systemic manifestations of prolonged malaise and fever while the chronic forms can develop from partial healing of acute circumstances, weak attacks or strong defenses. ${ }^{2}$

Infection is a process that involves the proliferation of microorganisms that cause the body's defense reaction, a process called inflammation. Inflammation is a vascular reaction which results in the delivery of fluids, dissolved substances and blood cells from the blood to the interstitial tissues of the injured or necrotic region. Acute inflammation is an immediate reaction to injury or cell death. The main signs of inflammation are dolor (pain), rubor (reddness), calor (heat), tumor (swelling) and functio laesa (alteration of function). To understand the difference between the types of inflammation, it is considered important to know and determine treatment therapy. ${ }^{3}$

Odontogenic infections are the most commonly encountered infections and a worldwide problem and hence, the main reason for seeking proper dental care. ${ }^{4}$ Odontogenic infections can develop from damaged teeth, trauma to the root area of teeth, pathogens in the mouth or the presence of inflammatory mediators that rapidly infiltrate nearby areas, such as submandibular trigonum or fossa canina. This can lead to the formation of fluids in soft tissues and abscess formation. In the case of a fossa canina abscess, formation of fluids can extend into the cranial region. Airway obstruction, periorbital abscess, and intracranial abscess are the most common and familiar symptoms when infection is increased. ${ }^{5}$

Odontogenic infections are common and can become a dangerous health problem when life-threatening complications occur. These infections can spread through the bone and periosteum against nearby structures increasing the risk of septicemia and death for affected patients. Fossa canina abscess is an odontogenic infection that can cause these life-threatening complications. The success of treatment requires early recognition, determination of etiological factors, and appropriate medical and surgical management. . $^{5,6}$

To perform treatment of odontogenic infections, the dentist should understand the terminology regarding infectious diseases and pathophysiology. Odontogenic infections stem from a variety of microbes, such as aerobic bacteria and facultative anaerobes. Anatomical factors play a key role in 
the presentation of bacterial infections that spread beyond the confines of the jaw. The spread of infection tends to follow the most opposite line determined by the bone and periosteum, muscle and fascia.?

Determining the severity of infection, evaluating the host defense, surgical management, medical support, administering antibiotics and periodic evaluation of patients are the main routes of odontogenic infection management. Three main factors to consider when determining the severity of infection in the head and neck are location anatomy, rate of progression, and airway compromise. $^{8}$

The spread of infection to the fossa canina usually originates from the maxillary canine or the upper premolar teeth, often seen above the buccinator muscle. This swelling removes the nasolabial folds, the space near the lower eyelid, and therefore early management is very important to avoid circular orbital infections. There is a risk of spread to the cranial through the external angle of the vein that can lead to thrombosis. ${ }^{8}$

\section{Case Report}

A 74-year-old man came with a swollen complaint on the right-side of his face. The complaint arose 2 weeks prior to his clinic visit and had spread under the eyes. The patient had a history of pain and fever. Physical examination of the face showed enlargement and swelling in the right cheek area figure 1 and 2. Panoramic radiographic images showed a radiolucent appearance of the tip of the apex at tooth 13 figure 3. Based on the results of the clinical and radiographic examinations, the patient was diagnosed with a fossa canina abscess. The treatment performed was an intraoral drainage incision at the apex area of tooth 13 figure 4 and 5 followed by continued antibiotic therapy.

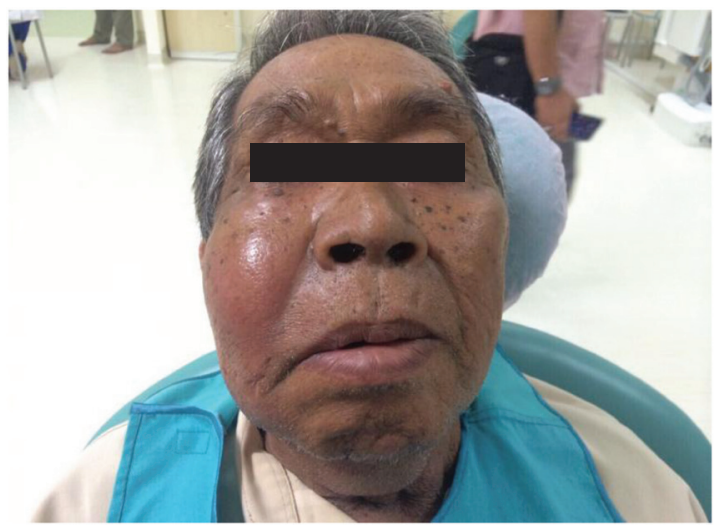

Figure 1 Extraoral picture

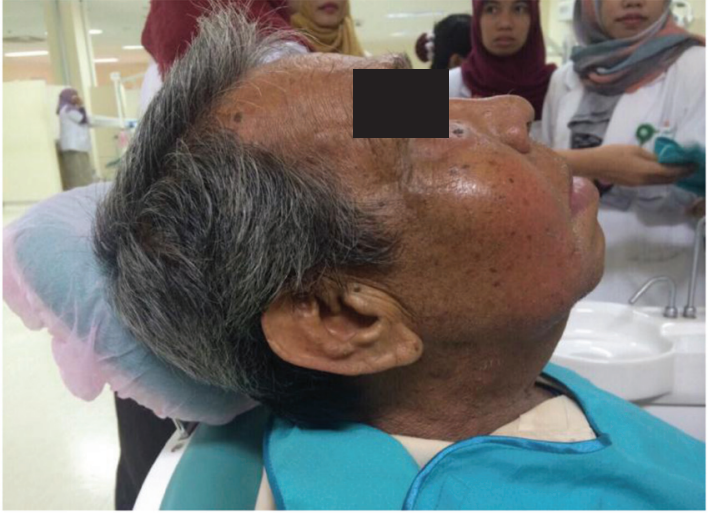

Figure 2 Side view

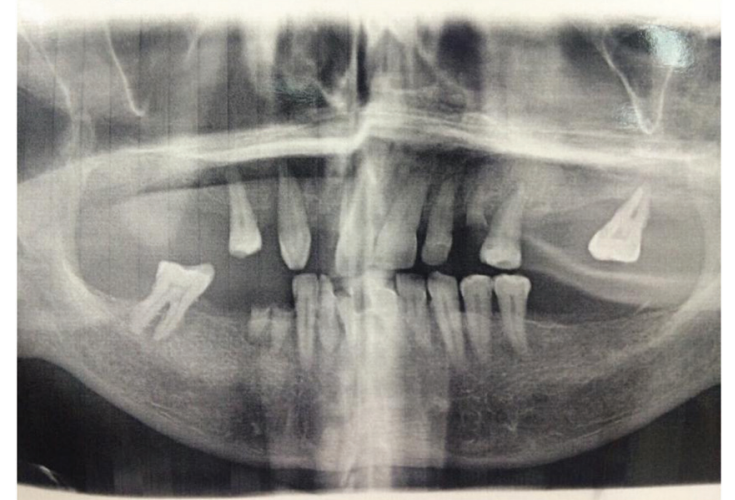

Figure 3 Panoramic radiographic features

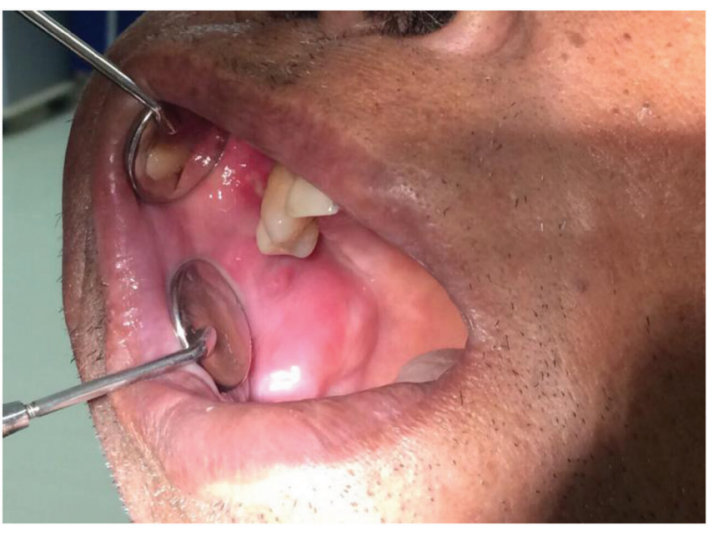

Figure 4 Intraoral clinical features

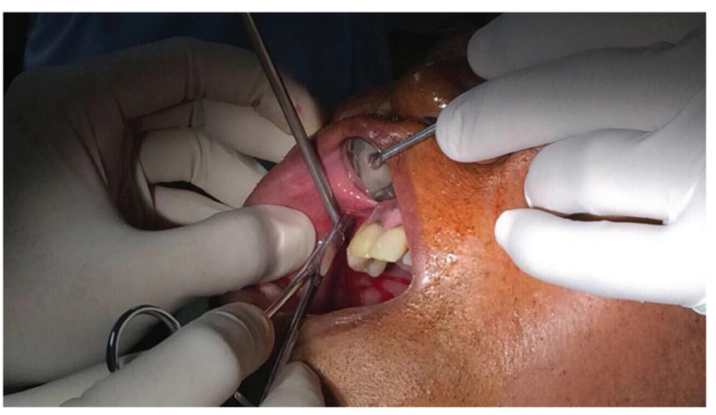

Figure 5 Intraoral drainage incision at the apex area of tooth 13 


\section{Discussion}

For all practical purposes, most infections originate in the mandible and jaw bone of the odontogenic source and are usually caused by periapical infections, periodontal infections, cysts, root residues, remaining infections, pericoronal pockets and others. $^{10}$

The causes and diagnoses of serious odontogenic infections, as well as their propensity to spread, has been described extensively in the literature. Fossa canina abscess is one type of odontogenic infection that has several trigger factors that may be associated with dental caries, periapical or periodontal abscess, pericoronitis, pulpitis, or osteitis. ${ }^{5,6}$

Orofacial infection can spread several ways, such as directly through the tissues, through the lymphatic system to the regional lymph nodes and into the bloodstream, or directly through the bloodstream. ${ }^{11}$ The spread of infection to the fossa canina usually arises from the maxillary canine teeth or other anterior teeth and the upper premolar teeth, often seen above the buccinator muscle. There is a risk the infection can spread to the cranial through the external angle of the vein and lead to thrombosis. ${ }^{7,11}$ This case shows swelling of the right cheek that extends under the eye. Panoramic radiographic results show radiolucent appearance in the apex region of tooth 13. Radiographic examination is an important component of dental management because periapical, occlusal and panoramic radiography can usually provide the necessary information. ${ }^{5}$

The results of a study conducted by Mathew et al., showed that $16 \%$ of odontogenic infections are in the fossa canina space and the main source these infections is infection of the pulp (70.8\%). The signs and symptoms that accompany this type of infection includes swelling, pain, trismus, fever, dysphagia, and pus expenditure. ${ }^{13}$

An odontogenic infection is a polymicrobial infection caused by a combination of anaerobic bacterial species, specifically facultative anaerobes and anaerobes. The most common microorganisms in dentoalveolar infections are streptococcus viridans and dominant anaerobes, such as anaerococcus, prevotella species and fusobacterium. ${ }^{7}$ The use of advanced non-culture methods have identified broader microorganisms, such as treponema species and gram-positive anaerobic rods, specifically Bulleidia extructa, Cryptobacterium curtum, and Mogibacterium timidum. ${ }^{8}$

Dental and medical practitioners have an important role in the management of odontogenic infections. They can treat patients with only antibiotics, administer the correct care or refer patients to oral and maxillofacial surgery specialists. Initially, antibiotics will work effectively but if the patient does not proceed to definitive management, the problem will reappear with an increase in the severity of the infection. Odontogenic infections can occur from dental care when practitioners try to maintain teeth with conservative management. ${ }^{7}$

Empirically, amoxicillin antibiotic therapy with potassium clavulanate and metronidazole, together with drainage incisions, provides excellent treatment outcomes for all patients. Beta-lactam antibiotics have been used to treat odontogenic infections because they are very effective against certain bacteria, cost effective, and have little side effects. Increased beta-lactam resistance, due to the emergence of organisms that produce beta-lactamase, has caused concern about the effectiveness of beta-lactam antibiotics in recent years. ${ }^{13}$

\section{Conclusion}

Odontogenic infections can be life-threatening with the formation of abscesses around the tissue structure. It is therefore important that dentists are aware of possible ways in which odontogenic infections can spread and know how to deal with such complications in case of an emergency. Fossa canina abscess is an odontogenic infection that can cause life-threatening complications. The success of treatment requires early recognition, determination of etiological factors, and appropriate medical and surgical management.

\section{Acknowledgment}

The author would like to thank the patient who has been willing to share his case for reported.

\section{Conflict of Interest}

The author report no conflict of interest.

\section{References}

1. Tamales D, Dewi N, Rosida L. Extract of haruan (channa striata) extract increasing reepithelialisation count in wound healing process on wistar rat's buccal mucosa. J Dentomaxillofac Sci 2016;1: 12-15.

2. Petersen GW. Oral surgery 1 ed. Philadelphia: WB Saunders Company; 1996. 191-197.

3. Fragiskos FD. The role of oral surgery in orthodontic treatment planning. Am J Orthodontic Dentofac Ortophedic 1956;42: 279-287.

4. Lopez-piriz L, Aguilar L, Gimenez JM. Management of odontogenic infection of pulpa and periodontal origin. Med oral patol oral cir bukal 2007;12: 154-159. 
5. Agacayak S, Atilgan S, Belgin G. Case report: canine fossa abscess; a rare etiological factor: the lower canine tooth. J Int Dent Med Res 2013;6: 36-39.

6. Veronez B, De-Matos, Monnazzi MS. Maxillofacial infection. A retrospective evaluation of eight years. Braz J Oral Sci 2014;13: 98-103.

7. Uluibau IC, Jaunay T, Goss AN. Severe odontogenic infections. Aust Dent J Med 2005;50: 74-80.

8. Ferrera PC, Busino LJ, Snyder HS. Uncommon complications of odontogenic infections. Am J Emerg Med 1996;14: 317-322.

9. Fischer, Bercu. Retention: a discussion of permanency of results in orthodontic practice. Am J Orthodontics and Oral Surg 1943;29: 5.

10. Lombardo GA, Karakourtis MH, White RPJ. The impact of clinical practice patterns on hospital charges for orthognathic surgery. Int J Adult Orthodon Orthognath Surg 1994;9: 251
11. Rishi KB, Parveen S, Shivani G, et al. A review of complications of odontogenic infections. Natl J Maxillofac Surg 2015;6: 136-143.

12. Gohel V. Manual of minor oral surgery for general dentist. British Dental Journal 2006;201: 735.

13. Mathew GC, Ranganathan LK, Gandhi S. Odontogenic maxillofacial space infections at a tertiary care center in North India: a five-year retrospective study. Int J Infec Diseases 2012;16: 296-302.

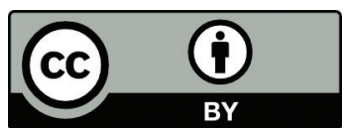

This work is licensed under a Creative Commons Attribution 